\title{
Constraint Solving via Fractional Edge Covers ${ }^{1}$
}

Martin Grohe, RWTH Aachen University, Lehrstuhl für Informatik 7, Aachen, Germany,

grohe@informatik.rwth-aachen.de

Dániel Marx, Computer and Automation Research Institute, Hungarian Academy of Sciences (MTA

SZTAKI), Budapest, Hungary, dmarx@cs.bme.hu. ${ }^{2}$

Many important combinatorial problems can be modeled as constraint satisfaction problems. Hence identifying polynomial-time solvable classes of constraint satisfaction problems has received a lot of attention. In this paper, we are interested in structural properties that can make the problem tractable. So far, the largest structural class that is known to be polynomial-time solvable is the class of bounded hypertree width instances introduced by Gottlob et al. [2002]. Here we identify a new class of polynomial-time solvable instances: those having bounded fractional edge cover number.

Combining hypertree width and fractional edge cover number, we then introduce the notion of fractional hypertree width. We prove that constraint satisfaction problems with bounded fractional hypertree width can be solved in polynomial time (provided that a the tree decomposition is given in the input). Together with a recent approximation algorithm for finding such decompositions [Marx 2010a], it follows that bounded fractional hypertree width is now the most general known structural property that guarantees polynomialtime solvability.

Categories and Subject Descriptors: F.2 [Theory of Computing]: Analysis of Algorithms and Problem Complexity; G.2.2 [Mathematics of Computing]: Discrete Mathematics-Graph Theory

General Terms: Algorithms

Additional Key Words and Phrases: constraint satisfaction, hypergraphs, hypertree width, fractional edge covers

\section{INTRODUCTION}

Constraint satisfaction problems form a large class of combinatorial problems that contains many important "real-world" problems. An instance of a constraint satisfaction problem consists of a set $V$ of variables, a domain $D$, and a set $C$ of constraints. For example, the domain may be $\{0,1\}$, and the constraints may be the clauses of a 3-CNF-formula. The objective is to assign values in $D$ to the variables in such a way that all constraints are satisfied. In general, constraint satisfaction problems are NP-hard; considerable efforts, both practical and theoretical, have been made to identify tractable classes of constraint satisfaction problems.

On the theoretical side, there are two main directions towards identifying polynomialtime solvable classes of constraint satisfaction problems. One is to restrict the constraint language, that is, the type of constraints that are allowed (see, for example, Bulatov 2006 . Bulatov 2011; Bulatov et al. 2001; Feder and Vardi 1998; Jeavons et al. 1997; Schaefer 1978). Formally, the constraint language can be described as a set of relations on the domain. The other direction is to restrict the structure induced by the constraints on the variables (see, for example, Cohen et al. 2008, Dalmau et al. 2002, Dechter and Pearl 1989; Freuder 1990, Kolaitis and Vardi 1998, Grohe et al. 2001; Grohe 2007]). The present work goes into this direction; our main contribution is the identification of a natural new class of structurally tractable constraint satisfaction problems.

The hypergraph of an instance $(V, D, C)$ has $V$ as its vertex set and for every constraint in $C$ a hyperedge that consists of all variables occurring in the constraint. For a class $\mathcal{H}$ of

\footnotetext{
${ }^{1}$ An extended abstract of the paper appeared in the Proceedings of the seventeenth annual ACM-SIAM Symposium on Discrete Algorithms (SODA 2006).

${ }^{2}$ Research supported by the European Research Council (ERC) grant "PARAMTIGHT: Parameterized complexity and the search for tight complexity results," reference 280152.
} 
hypergraphs, we let $\operatorname{CsP}(\mathcal{H})$ be the class of all instances whose hypergraph is contained in $\mathcal{H}$. The central question is for which classes $\mathcal{H}$ of hypergraphs the problem $\operatorname{Csp}(\mathcal{H})$ is tractable. Most recently, this question has been studied in Chen and Dalmau 2005: Cohen et al. 2008; Marx 2011; Marx 2010b; Gottlob et al. 2005]. It is worth pointing out that the corresponding question for the graphs (instead of hypergraphs) of instances, in which two variables are incident if they appear together in a constraint, has been completely answered in Grohe 2007: Grohe et al. 2001] (under the complexity theoretic assumption FPT $\neq \mathrm{W}[1]$ ): For a class $\mathcal{G}$ of graphs, the corresponding problem $\operatorname{CsP}(\mathcal{G})$ is in polynomial time if and only if $\mathcal{G}$ has bounded tree width. This can be generalized to $\operatorname{Csp}(\mathcal{H})$ for classes $\mathcal{H}$ of hypergraphs of bounded hyperedge size (that is, classes $\mathcal{H}$ for which $\max \{|e| \mid \exists H=(V, E) \in \mathcal{H}: e \in E\}$ exists). It follows easily from the results of Grohe 2007: Grohe et al. 2001 that for all classes $\mathcal{H}$ of bounded hyperedge size,

$$
\operatorname{CsP}(\mathcal{H}) \in \operatorname{PTIME} \Longleftrightarrow \mathcal{H} \text { has bounded tree width }
$$

(under the assumption FPT $\neq \mathrm{W}[1]$ ).

It is known that (1) does not generalize to arbitrary classes $\mathcal{H}$ of hypergraphs (we will give a very simple counterexample in Section 22). The largest known family of classes of hypergraphs for which $\operatorname{CsP}(\mathcal{H})$ is in PTIME consists of all classes of bounded hypertree width Gottlob et al. 2002, Gottlob et al. 2003, Gottlob et al. 2000. Hypertree width is a hypergraph invariant that generalizes acyclicity Berge 1976; Fagin 1983: Yannakakis 1981]. It is a very robust invariant; up to a constant factor it coincides with a number of other natural invariants that measure the global connectivity of a hypergraph [Adler et al. 2007. On classes of bounded hyperedge size, bounded hypertree width coincides with bounded tree width, but in general it does not. It has been asked in Chen and Dalmau 2005 Cohen et al. 2008: Gottlob et al. 2005, Grohe 2007 whether there are classes $\mathcal{H}$ of unbounded hypertree width such that $\operatorname{CsP}(\mathcal{H}) \in$ PTIME. We give an affirmative answer to this question.

Our key result states that $\operatorname{Csp}(\mathcal{H}) \in$ PTIME for all classes $\mathcal{H}$ of bounded fractional edge cover number. A fractional edge cover of a hypergraph $H=(V, E)$ is a mapping $x: E \rightarrow[0, \infty)$ such that $\sum_{e \in E, v \in e} x(e) \geq 1$ for all $v \in V$. The number $\sum_{e \in E} x(e)$ is the weight of $x$. The fractional edge cover number $\rho^{*}(H)$ of $H$ is the minimum of the weights of all fractional edge covers of $H$. It follows from standard linear programming results that this minimum exists and is rational. Furthermore, it is easy to construct classes $\mathcal{H}$ of hypergraphs that have bounded fractional edge cover number and unbounded hypertree width (see Example 4.2).

We then start a more systematic investigation of the interaction between fractional covers and hypertree width. We propose a new hypergraph invariant, the fractional hypertree width, which generalizes both the hypertree width and fractional edge cover number in a natural way. Fractional hypertree width is an interesting hybrid of the "continuous" fractional edge cover number and the "discrete" hypertree width. We show that it has properties that are similar to the nice properties of hypertree width. In particular, we give an approximative game characterization of fractional hypertree width similar to the characterization of tree width by the "cops and robber" game Seymour and Thomas 1993. Furthermore, we prove that for classes $\mathcal{H}$ of bounded fractional hypertree width, the problem $\operatorname{CsP}(\mathcal{H})$ can be solved in polynomial time provided that a fractional hypertree decomposition of the underlying hypergraph is given together with the input instance. We do not know if for every fixed $k$ there is a polynomial-time algorithm for finding a fractional hypertree decomposition of width $k$. However, a recent result Marx 2010a shows that we can find an approximate decomposition whose width is bounded by a (cubic) function of the fractional hypertree width. This is sufficient to show that $\operatorname{CsP}(\mathcal{H})$ is polynomial-time solvable for classes $\mathcal{H}$ of bounded fractional hypertree width, even if no decomposition is given in the input. Therefore, bounded fractional hypertree width is the so far most general hypergraph property that 


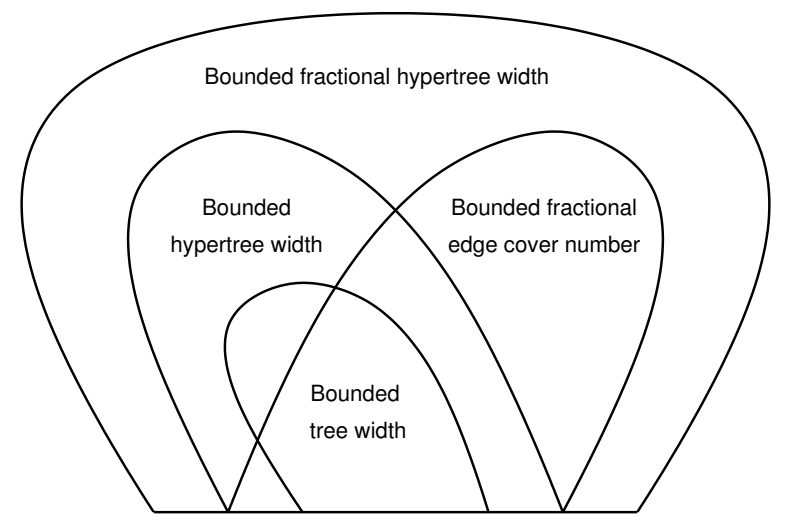

Fig. 1. Hypergraph properties that make CSP polynomial-time solvable.

makes $\operatorname{CsP}(\mathcal{H})$ polynomial-time solvable. Note that this property is strictly more general than bounded hypertree width and bounded fractional edge cover number (see Figure 1).

For classes $\mathcal{H}$ of hypergraphs of bounded fractional hypertree width, we also show that all solutions of an instance of $\operatorname{CsP}(\mathcal{H})$ can be computed by a polynomial delay algorithm. Closely related to the problem of computing all solutions of a CsP-instance is the problem of evaluating a conjunctive query against a relational database. We show that conjunctive queries whose underlying hypergraph is in $\mathcal{H}$ can be evaluated by a polynomial delay algorithm as well. Finally, we look at the homomorphism problem and the embedding problem for relational structures. The homomorphism problem is known to be equivalent to the CSP Feder and Vardi 1998 and hence can be solved in polynomial time if the left-hand side structure has an underlying hypergraph in a class $\mathcal{H}$ of bounded fractional hypertree width. This implies that the corresponding embedding problem, parameterized by the size of the universe of the left-hand side structure, is fixed-parameter tractable. Recall that a problem is fixed-parameter tractable (FPT) by some parameter $k$ if the problem can be solved in time $f(k) \cdot n^{O(1)}$ for a computable function $f$ depending only on $k$. In particular, if a problem is polynomial-time solvable, then it is fixed-parameter tractable (with any parameter $k$ ).

Follow up work. Let us briefly discuss how the ideas presented in the conference version of this paper Grohe and Marx 2006] influenced later work. The conference version of this paper posed as an open question whether for every fixed $k$ there is a polynomial-time algorithm that, given a hypergraph with fractional hypertree width $k$, finds a fractional hypertree decomposition having width $k$ or, at least, having width bounded by a function of $k$. This question has been partially resolved by the algorithm of Marx 2010a that finds a fractional hypertree decomposition of width $O\left(k^{3}\right)$ if a decomposition of width $k$ exists. This algorithm makes the results of the present paper stronger, as the polynomial-time algorithms for CSPs with bounded fractional hypertree width no longer need to assume that a decomposition is given in the input (see Section 4.3). The problem of finding a decomposition of width exactly $k$, if such a decomposition exists, is still open. In the special case of hypergraphs whose incidence graphs are planar, a constant-factor approximation of fractional hypertree width can be found by exploiting the fact that for such graphs fractional hypertree width and the tree width of the incidence graph can differ only by at most a constant factor Fomin et al. 2009].

A core combinatorial idea of the present paper is that Shearer's Lemma gives an upper bound on the number of solutions of a CSP instance and we use this bound for the subproblems corresponding to the bags of a fractional hypertree decomposition. This upper bound has been subsequently used by Atserias et al. [2008] in the context of database 
queries, where it is additionally shown that a linear programming duality argument implies the tightness of this bound. We repeat this argument here as Theorem 3.7. An optimal query evaluation algorithm matching this tight bound was given by Ngo et al. [2012]. The bound was generalized to the context of conjunctive queries with functional dependencies by Gottlob et al. [2012].

We show that if $\mathcal{H}$ is a class of hypergraphs with bounded fractional hypertree width, then $\operatorname{Csp}(\mathcal{H})$ is fixed-parameter tractable parameterized by the number of variables and, in fact, polynomial-time solvable (using the algorithm of [Marx 2010a] for finding decompositions). It is a natural question if there are more general fixed-parameter tractable or polynomialtime solvable classes of hypergraphs. Very recently, Marx [2010b gave a strictly more general such class by introducing the notion of submodular width and showing that $\operatorname{CsP}(\mathcal{H})$ is fixedparameter tractable for classes $\mathcal{H}$ with bounded submodular width. Furthermore, it was shown in Marx 2010b] that there are no classes $\mathcal{H}$ with unbounded submodular width that make $\operatorname{Csp}(\mathcal{H})$ fixed-parameter tractable (the proof uses a complexity-theoretic assumption called Exponential Time Hypothesis Impagliazzo et al. 2001). However, with respect to polynomial-time solvability, bounded fractional hypertree width is still the most general known tractability condition and it is an open question whether there are classes $\mathcal{H}$ with unbounded fractional hypertree width such that $\operatorname{CsP}(\mathcal{H})$ is polynomial-time solvable.

\section{PRELIMINARIES}

\subsection{Hypergraphs}

A hypergraph is a pair $H=(V(H), E(H))$, consisting of a set $V(H)$ of vertices and a set $E(H)$ of nonempty subsets of $V(H)$, the hyperedges of $H$. We always assume that hypergraphs have no isolated vertices, that is, for every $v \in V(H)$ there exists at least one $e \in E(H)$ such that $v \in e$.

For a hypergraph $H$ and a set $X \subseteq V(H)$, the subhypergraph of $H$ induced by $X$ is the hypergraph $H[X]=(X,\{e \cap X \mid e \in \bar{E}(H)$ with $e \cap X \neq \emptyset\})$. We let $H \backslash X=H[V(H) \backslash X]$. The primal graph of a hypergraph $H$ is the graph

$$
\begin{array}{r}
\underline{H}=(V(H),\{\{v, w\} \mid v \neq w, \text { there exists an } \\
e \in E(H) \text { such that }\{v, w\} \subseteq e\}) .
\end{array}
$$

A hypergraph $H$ is connected if $\underline{H}$ is connected. A set $C \subseteq V(H)$ is connected (in $H$ ) if the induced subhypergraph $H[C]$ is connected, and a connected component of $H$ is a maximal connected subset of $V(H)$. A sequence of vertices of $H$ is a path of $H$ if it is a path of $\underline{H}$.

A tree decomposition of a hypergraph $H$ is a tuple $\left(T,\left(B_{t}\right)_{t \in V(T)}\right)$, where $T$ is a tree and $\left(B_{t}\right)_{t \in V(T)}$ a family of subsets of $V(H)$ such that for each $e \in E(H)$ there is a node $t \in V(T)$ such that $e \subseteq B_{t}$, and for each $v \in V(H)$ the set $\left\{t \in V(T) \mid v \in B_{t}\right\}$ is connected in $T$. The sets $B_{t}$ are called the bags of the decomposition. The width of a tree-decomposition $\left(T,\left(B_{t}\right)_{t \in V(T)}\right)$ is $\max \left\{\left|B_{t}\right| \mid t \in V(t)\right\}-1$. The tree width $\operatorname{tw}(H)$ of a hypergraph $H$ is the minimum of the widths of all tree-decompositions of $H$. It is easy to see that $\operatorname{tw}(H)=\operatorname{tw}(\underline{H})$ for all $H$.

It will be convenient for us to view the trees in tree-decompositions as being rooted and directed from the root to the leaves. For a node $t$ in a (rooted) tree $T=(V(T), E(T)$ ), we let $T_{t}$ be the subtree rooted at $t$, that is, the induced subtree of $T$ whose vertex set is the set of all vertices reachable from $t$.

We say that a class $\mathcal{H}$ of hypergraphs is of bounded tree width if there is a $k$ such that $\operatorname{tw}(H) \leq k$ for all $H \in \mathcal{H}$. We use a similar terminology for other hypergraph invariants.

\subsection{Constraint satisfaction problems}

A CSP instance is a triple $I=(V, D, C)$, where $V$ is a set of variables, $D$ is a set called the domain, and $C$ is a set of constraints of the form $\left\langle\left(v_{1}, \ldots, v_{k}\right), R\right\rangle$, where $k \geq 1$ and $R$ is a 
$k$-ary relation on $D$. A solution to the instance $I$ is an assignment $\alpha: V \rightarrow D$ such that for all constraints $\left\langle\left(v_{1}, \ldots, v_{k}\right), R\right\rangle$ in $C$ we have $\left(\alpha\left(v_{1}\right), \ldots, \alpha\left(v_{k}\right)\right) \in R$.

Constraints are specified by explicitly enumerating all possible combinations of values for the variables, that is, all tuples in the relation $R$. Consequently, we define the size of a constraint $c=\left\langle\left(v_{1}, \ldots, v_{k}\right), R\right\rangle \in C$ to be the number $\|c\|=k+k \cdot|R|$. The size of an instance $I=(V, D, C)$ is the number $\|I\|=|V|+|D|+\sum_{c \in C}\|c\|$. Of course, there is no need to store a constraint relation repeatedly if it occurs in several constraints, but this only changes the size by a polynomial factor.

Let us make a few remarks about this explicit representation of the constraints. There are important special cases of constraint satisfaction problems where the constraints are stored implicitly, which may make the representation exponentially more succinct. Examples are Boolean satisfiability, where the constraint relations are given implicitly by the clauses of a formula in conjunctive normal form, or systems of arithmetic (in)equalities, where the constraints are given implicitly by the (in)equalities. However, our representation is the standard "generic" representation of constraint satisfaction problems in artificial intelligence (see, for example, Dechter 2003]). An important application where the constraints are always given in explicit form is the conjunctive query containment problem, which plays a crucial role in database query optimization. Kolaitis and Vardi Kolaitis and Vardi 1998 observed that it can be represented as a constraint satisfaction problem, and the constraint relations are given explicitly as part of one of the input queries. A related problem from database systems is the problem of evaluating conjunctive queries (cf. Theorem 4.14). Here the constraint relations represent the tables of a relational database, and again they are given in explicit form. The problem of characterizing the tractable structural restrictions of CSP has also been studied for other representations of the instances: one can consider more succinct representations such as disjunctive formulas or decision diagrams Chen and Grohe 2010] or less succinct representations such as truth tables Marx 2011. As the choice of representation influences the size of the input and the running time is expressed as a function of the input size, the choice of representation influences the complexity of the problem and the exact tractability criterion.

Observe that there is a polynomial-time algorithm deciding whether a given assignment for an instance is a solution.

The hypergraph of the CSP instance $I=(V, D, C)$ is the hypergraph $H_{I}$ with vertex set $V$ and a hyperedge $\left\{v_{1}, \ldots, v_{k}\right\}$ for all constraints $\left\langle\left(v_{1}, \ldots, v_{k}\right), R\right\rangle$ in $C$. For every class $\mathcal{H}$, we consider the following decision problem:

\section{$\operatorname{CsP}(\mathcal{H})$}

Instance: A CSP instance $I$ with $H_{I} \in \mathcal{H}$.

Problem: Decide if $I$ has a solution.

If the class $\mathcal{H}$ is not polynomial-time decidable, we view this as a promise problem, that is, we assume that we are only given instances $I$ with $H_{I} \in \mathcal{H}$, and we are only interested in algorithms that work correctly and efficiently on such instances.

We close this section with a simple example of a class of hypergraphs of unbounded tree width such that $\operatorname{CsP}(\mathcal{H})$ is tractable.

Example 2.1. Let $\mathcal{H}$ be that class of all hypergraphs $H$ that have a hyperedge that contains all vertices, that is, $V(H) \in E(H)$. Clearly, $\mathcal{H}$ has unbounded tree width, because the hypergraph $(V,\{V\})$ has tree width $|V|-1$. We claim that $\operatorname{CsP}(\mathcal{H}) \in \operatorname{PTIME}$.

To see this, let $I=(V, D, C)$ be an instance of $\operatorname{Csp}(\mathcal{H})$. Let $\left\langle\left(v_{1}, \ldots, v_{k}\right), R\right\rangle$ be a constraint in $C$ with $\left\{v_{1}, \ldots, v_{k}\right\}=V$. Such a constraint exists because $H_{I} \in \mathcal{H}$. Each tuple $\bar{d}=\left(d_{1}, \ldots, d_{k}\right) \in R$ completely specifies an assignment $\alpha_{\bar{d}}$ defined by $\alpha_{\bar{d}}\left(v_{i}\right)=d_{i}$ for $1 \leq i \leq k$. If for some $i, j$ we have $v_{i}=v_{j}$, but $d_{i} \neq d_{j}$, we leave $\alpha_{\bar{d}}$ undefined. 
Observe that $I$ is satisfiable if and only if there is a tuple $\bar{d} \in R$ such that $\alpha_{\bar{d}}$ is (welldefined and) a solution for $I$. As $|R| \leq\|I\|$, this can be checked in polynomial time.

\section{A POLYNOMIAL-TIME ALGORITHM FOR CSPS WITH BOUNDED FRACTIONAL COVER NUMBER}

In this section we prove that if the hypergraph $H_{I}$ of a CSP instance $I$ has fractional edge cover number $\rho^{*}\left(H_{I}\right)$, then it can be decided in $\|I\|^{\rho^{*}\left(H_{I}\right)+O(1)}$ time whether $I$ has a solution. Thus if $\mathcal{H}$ is a class of hypergraphs with bounded fractional edge cover number (that is, there is a constant $r$ such that $\rho^{*}(H) \leq r$ for every $\left.H \in \mathcal{H}\right)$, then $\operatorname{Csp}(\mathcal{H}) \in \operatorname{PTIME}$. Actually, we prove a stronger result: A CSP instance $I$ has at most $\|I\|^{\rho^{*}\left(H_{I}\right)}$ solutions and all the solutions can be enumerated in time $\|I\| \rho^{*}\left(H_{I}\right)+O(1)$.

The proof relies on a combinatorial lemma known as Shearer's Lemma. We use Shearer's Lemma to bound the number of solutions of a CSP instance; our argument resembles an argument that Friedgut and Kahn Friedgut and Kahn 1998 used to bound the number of subhypergraphs of a certain isomorphism type in a hypergraph. The second author applied similar ideas in a completely different algorithmic context [Marx 2008].

The entropy of a random variable $X$ with range $U$ is

$$
h[X]:=-\sum_{x \in U} \operatorname{Pr}(X=x) \log \operatorname{Pr}(X=x)
$$

Shearer's lemma gives an upper bound of a distribution on a product space in terms of its marginal distributions.

Lemma 3.1 (SheareR's Lemma CHUng ET Al. 1986]). Let $X=\left(X_{i} \mid i \in I\right)$ be a random variable, and let $A_{j}$, for $j \in[m]$, be (not necessarily distinct) subsets of the index set $I$ such that each $i \in I$ appears in at least $q$ of the sets $A_{j}$. For every $B \subseteq I$, let $X_{B}=\left(X_{i} \mid i \in B\right)$. Then

$$
\sum_{j=1}^{m} h\left[X_{A_{j}}\right] \geq q \cdot h[X] .
$$

Lemma 3.1 is easy to see in the special case when $q=1$ and $\left\{A_{1}, \ldots, A_{p}\right\}$ is a partition of $V$. The proof of the general case in Chung et al. 1986 is based on the submodularity of entropy. See also Rhadakrishnan for a simple proof.

LEMMA 3.2. If $I=(V, D, C)$ is a CSP instance where every constraint relation contains at most $N$ tuples, then $I$ has at most $N^{\rho^{*}\left(H_{I}\right)} \leq\|I\|^{\rho^{*}\left(H_{I}\right)}$ solutions.

Proof. Let $x$ be a fractional edge cover of $H_{I}$ with $\sum_{e \in E\left(H_{I}\right)} x(e)=\rho^{*}\left(H_{I}\right)$; it follows from the standard results of linear programming that such an $x$ exists with rational values. Let $p_{e}$ and $q$ be nonnegative integers such that $x(e)=p_{e} / q$. Let $m=\sum_{e \in E\left(H_{I}\right)} p_{e}$, and let $A_{1}, \ldots, A_{m}$ be a sequence of subsets of $V$ that contains precisely $p_{e}$ copies of the set $e$, for all $e \in E\left(H_{I}\right)$. Then every variable $v \in V$ is contained in at least

$$
\sum_{e \in E\left(H_{I}\right): v \in e} p_{e}=q \cdot \sum_{e \in E\left(H_{I}\right): v \in e} x(e) \geq q
$$

of the sets $A_{i}$ (as $x$ is a fractional edge cover). Let $X=\left(X_{v} \mid v \in V\right)$ be uniformly distributed on the solutions of $I$, which we assume to be non-empty as otherwise the claim is obvious. That is, if we denote by $S$ the number of solutions of $I$, then we have $\operatorname{Pr}(X=\alpha)=1 / S$ for every solution $\alpha$ of $I$. Then $h[X]=\log S$. We apply Shearer's Lemma to the random variable $X$ and the sequence $A_{1}, \ldots, A_{m}$ of subsets of $V$. Assume that $A_{i}$ corresponds to some constraint $\left\langle\left(v_{1}^{\prime}, \ldots, v_{k}^{\prime}\right), R\right\rangle$. Then the marginal distribution of $X$ on $\left(v_{1}^{\prime}, \ldots, v_{k}^{\prime}\right)$ is 0 
on all tuples not in $R$. Hence the entropy of $X_{A_{i}}$ is is bounded by the entropy of the uniform distribution on the tuples in $R$, that is, $h\left[X_{A_{i}}\right] \leq \log N$. Thus by Shearer's Lemma, we have

$$
\sum_{e \in E\left(H_{I}\right)} p_{e} \cdot \log N \geq \sum_{e \in E\left(H_{I}\right)} p_{e} \cdot h\left[X_{e}\right]=\sum_{i=1}^{m} h\left[X_{A_{i}}\right] \geq q \cdot h[X]=q \cdot \log S .
$$

It follows that

$$
S \leq 2^{\sum_{e \in E\left(H_{I}\right)}\left(p_{e} / q\right) \cdot \log N}=2^{\rho^{*}\left(H_{I}\right) \cdot \log N}=N^{\rho^{*}\left(H_{I}\right)} .
$$

We would like to turn the upper bound of Lemma 3.2 into an algorithm enumerating all the solutions, but the proof of Shearer's Lemma is not algorithmic. However, a very simple algorithm can enumerate the solutions, and Lemma 3.2 can be used to bound the running time of this algorithm. Starting with a trivial subproblem consisting only of a single variable, the algorithm enumerates all the solutions for larger and larger subproblems by adding one variable at a time. To define these subproblems, we need the following definitions:

Definition 3.3. Let $R$ be an $r$-ary relation over a set $D$. For $1 \leq i_{1}<\cdots<i_{\ell} \leq r$, the projection of $R$ onto the components $i_{1}, \ldots, i_{\ell}$ is the relation $R^{\mid i_{1}, \ldots, \bar{i}_{\ell}}$ which contains an $\ell$-tuple $\left(d_{1}^{\prime}, \ldots, d_{\ell}^{\prime}\right) \in D^{\ell}$ if and only if there is a $k$-tuple $\left(d_{1}, \ldots, d_{k}\right) \in R$ such that $d_{j}^{\prime}=d_{i_{j}}$ for $1 \leq j \leq \ell$.

Intuitively, a tuple is in $R^{\mid i_{1}, \ldots, i_{\ell}}$ if it can be extended into a tuple in $R$.

Definition 3.4. Let $I=(V, D, C)$ be a $C S P$ instance and let $V^{\prime} \subseteq V$ be a nonempty subset of variables. The CSP instance $I\left[V^{\prime}\right]$ induced by $V^{\prime}$ is $I^{\prime}=\left(\overline{V^{\prime}}, D, C^{\prime}\right)$, where $C^{\prime}$ is defined in the following way: for each constraint $c=\left\langle\left(v_{1}, \ldots, v_{k}\right), R\right\rangle$ having at least one variable in $V^{\prime}$, there is a corresponding constraint $c^{\prime}$ in $C^{\prime}$. Suppose that $v_{i_{1}}, \ldots, v_{i_{\ell}}$ are the variables among $v_{1}, \ldots, v_{k}$ that are in $V^{\prime}$. Then the constraint $c^{\prime}$ is defined as $\left\langle\left(v_{i_{1}}, \ldots, v_{i_{\ell}}\right), R^{\mid i_{1}, \ldots, i_{\ell}}\right\rangle$, that is, the relation is the projection of $R$ onto the components $i_{1}, \ldots, i_{\ell}$.

Thus an assignment $\alpha$ on $V^{\prime}$ satisfies $I\left[V^{\prime}\right]$ if for each constraint $c$ of $I$, there is an assignment extending $\alpha$ that satisfies $c$ (however, it is not necessarily true that there is an assignment extending $\alpha$ that satisfies every constraint of $I$ simultaneously). Note that that the hypergraph of the induced instance $I\left[V^{\prime}\right]$ is exactly the induced subhypergraph $H_{I}\left[V^{\prime}\right]$.

THEOREM 3.5. The solutions of a CSP instance $I$ can be enumerated in time $\|I\| \rho^{*}\left(H_{I}\right)+O(1)$.

Proof. Let $V=\left\{v_{1}, \ldots, v_{n}\right\}$ be an arbitrary ordering of the variables of $I$ and let $V_{i}$ be the subset $\left\{v_{1}, \ldots, v_{i}\right\}$. For $i=1,2, \ldots, n$, the algorithm creates a list $L_{i}$ containing the solutions of $I\left[V_{i}\right]$. Since $I\left[V_{n}\right]=I$, the list $L_{n}$ is exactly what we want.

For $i=1$, the instance $I\left[V_{i}\right]$ has at most $|D|$ solutions, hence the list $L_{i}$ is easy to construct. Notice that a solution of $I\left[V_{i+1}\right]$ induces a solution of $I\left[V_{i}\right]$. Therefore, the list $L_{i+1}$ can be constructed by considering the solutions in $L_{i}$, extending them to the variable $v_{i+1}$ in all the $|D|$ possible ways, and checking whether this assignment is a solution of $I\left[V_{i+1}\right]$. Clearly, this can be done in $\left|L_{i}\right| \cdot|D| \cdot\left\|I\left[V_{i+1}\right]\right\|^{O(1)}=\left|L_{i}\right| \cdot\|I\|^{O(1)}$ time. By repeating this procedure for $i=1,2, \ldots, n-1$, the list $L_{n}$ can be constructed.

The total running time of the algorithm can be bounded by $\sum_{i=1}^{n-1}\left|L_{i}\right| \cdot\|I\| \|^{O(1)}$. Observe that $\rho^{*}\left(H_{I\left[V_{i}\right]}\right) \leq \rho^{*}\left(H_{I}\right): H_{I\left[V_{i}\right]}$ is the subhypergraph of $H_{I}$ induced by $V_{i}$, thus any fractional cover of the hypergraph of $I$ gives a fractional cover of $I\left[V_{i}\right]$ (for every edge $e \in E\left(H_{I\left[V_{i}\right]}\right)$, we set the weight of $e$ to be the sum of the weight of the edges $e^{\prime} \in E\left(H_{I}\right)$ 
with $e^{\prime} \cap V_{i}=e$ ). Therefore, by Lemma $3.2,\left|L_{i}\right| \leq\|I\|^{\rho^{*}\left(H_{I}\right)}$, and it follows that the total running time is $\|I\| \rho^{*}\left(H_{I}\right)+O(1)$.

We note that the algorithm of Theorem 3.5 does not actually need a fractional edge cover: the fact that the hypergraph has small fractional edge cover number is used only in proving the time bound of the algorithm. By a significantly more complicated algorithm, Ngo et al. Ngo et al. 2012 improved Theorem 3.5 by removing the $O(1)$ term from the exponent.

COROLlary 3.6. Let $\mathcal{H}$ be a class of hypergraphs of bounded fractional edge cover number. Then $\operatorname{CsP}(\mathcal{H})$ is in polynomial time.

We conclude this section by pointing out that Lemma 3.2 is tight: there are arbitrarily large instances $I$ where every constraint relation contains at most $N$ tuples and the number of solutions is exactly $N^{\rho^{*}\left(H_{I}\right)}$. A similar proof appeared first in Atserias et al. 2008 in the context of database queries, but we restate it here in the language of CSPs for the convenience of the reader.

TheOREM 3.7. Let $H$ be a hypergraph. For every $N_{0} \geq 1$, there is a CSP instance $I=(V, D, C)$ with hypergraph $H$ where every constraint relation contains at most $N \geq N_{0}$ tuples and $I$ has at least $N^{\rho^{*}(H)}$ solutions.

Proof. A fractional independent set of hypergraph $H$ is an assignment $y: V(H) \rightarrow[0,1]$ such that $\sum_{v \in e} y(v) \leq 1$ for every $e \in E(H)$. The weight of $y$ is $\sum_{v \in V(H)} y(H)$. The fractional independent set number $\alpha^{*}(H)$ is the maximum weight of a fractional independent set of $H$. It is a well-known consequence of linear-programming duality that $\alpha^{*}(H)=\rho^{*}(H)$ for every hypergraph $H$, since the two values can be expressed by a pair of primal and dual linear programs [Schrijver 2003, Section 30.10].

Let $y$ be a fractional independent set of weight $\alpha^{*}(H)$. By standard results of linear programming, we can assume that $y$ is rational, that is, there is an integer $q \geq 1$ such that for every $v \in V(H), y(v)=p_{v} / q$ for some nonnegative integer $p_{v}$. We define a CSP instance $I=(V, D, C)$ with $V=V(H)$ and $D=\left[N_{0}^{q}\right]$ such that for every $e \in E(H)$ where $e=\left\{v_{1}, \ldots, v_{r}\right\}$, there is a constraint $\left\langle\left(v_{1}, \ldots, v_{r}\right), R_{e}\right\rangle$ with

$$
R_{e}=\left\{\left(a_{1}, \ldots, a_{r}\right) \mid a_{i} \in\left[N_{0}^{p_{v}}\right] \text { for every } 1 \leq i \leq r\right\} .
$$

Let $N=N_{0}^{q}$. We claim that $R_{e}$ contains at most $N$ tuples. Indeed, the number of tuples in $R_{e}$ is exactly

$$
\prod_{v \in e} N_{0}^{p_{v}}=N_{0}^{\sum_{v \in e} p_{v}}=N_{0}^{q \cdot \sum_{v \in e} p_{v} / q}=\left(N_{0}^{q}\right)^{\sum_{v \in e} y(v)} \leq N_{0}^{q}=N,
$$

since $y$ is a fractional independent set. Observe that $\alpha: V(H) \rightarrow D$ is a solution if and only if $\alpha(v) \in\left[N_{0}^{p_{v}}\right]$ for every $v \in V(H)$. Hence the number of solutions is exactly

$$
\prod_{v \in V(H)} N_{0}^{p_{v}}=N_{0}^{\sum_{v \in V(H)} p_{v}}=N_{0}^{q \cdot \sum_{v \in V(H)} p_{v} / q}=\left(N_{0}^{q}\right)^{\alpha^{*}(H)}=N^{\alpha^{*}(H)}=N^{\rho^{*}(H)},
$$

as required.

The significance of this result is that it shows that there is no "better" measure than fractional edge cover number that guarantees a polynomial bound on the number of solutions, in the following formal sense. Let $w(H)$ be a width measure that guarantees a polynomial bound: that is, if $I$ is a CSP instance where every relation has at most $N$ tuples, then $I$ has at most $N^{w(H)}$ solutions for some function $f$. Then by Theorem 3.7, we have $\rho^{*}(H) \leq w(H)$. This means the upper bound on the number of solutions given by $w(H)$ already follows from the bound given by Lemma 3.2 and hence $\rho^{*}(H)$ can be considered a stronger measure. 


\section{FRACTIONAL HYPERTREE DECOMPOSITIONS}

Let $H$ be a hypergraph. A generalized hypertree decomposition of $H$ Gottlob et al. 2002 is a triple $\left(T,\left(B_{t}\right)_{t \in V(T)},\left(C_{t}\right)_{t \in V(T)}\right)$, where $\left(T,\left(B_{t}\right)_{t \in V(T)}\right)$ is a tree decomposition of $H$ and $\left(C_{t}\right)_{t \in V(T)}$ is a family of subsets of $E(H)$ such that for every $t \in V(T)$ we have $B_{t} \subseteq \bigcup C_{t}$. Here $\bigcup C_{t}$ denotes the union of the sets (hyperedges) in $C_{t}$, that is, the set $\{v \in V(H) \mid$ $\left.\exists e \in C_{t}: v \in e\right\}$. We call the sets $B_{t}$ the bags of the decomposition and the sets $C_{t}$ the guards. The width of $\left(T,\left(B_{t}\right)_{t \in V(T)},\left(C_{t}\right)_{t \in V(T)}\right)$ is $\max \left\{\left|C_{t}\right| \mid t \in V(T)\right\}$. The generalized hypertree width $\operatorname{ghw}(H)$ of $H$ is the minimum of the widths of the generalized hypertree decompositions of $H$. The edge cover number $\rho(H)$ of a hypergraph is the minimum number of edges needed to cover all vertices; it is easy to see that $\rho(H) \geq \rho^{*}(H)$. Observe that the size of $C_{t}$ has to be at least $\rho\left(H\left[B_{t}\right]\right)$ and, conversely, for a given $B_{t}$ there is always a suitable guard $C_{t}$ of size $\rho\left(H\left[B_{t}\right]\right)$. Therefore, $\operatorname{ghw}(H) \leq r$ if there is a tree decomposition where $\rho\left(H\left[B_{t}\right]\right) \leq r$ for every $t \in V(T)$.

For the sake of completeness, let us mention that a hypertree decomposition of $H$ is a generalized hypertree decomposition $\left(T,\left(B_{t}\right)_{t \in V(T)},\left(C_{t}\right)_{t \in V(T)}\right)$ that satisfies the following additional special condition: $\left(\bigcup C_{t}\right) \cap \bigcup_{u \in V\left(T_{t}\right)} B_{u} \subseteq B_{t}$ for all $t \in V(T)$. Recall that $T_{t}$ denotes the subtree of the $T$ with root $t$. The hypertree width $\mathrm{hw}(H)$ of $H$ is the minimum of the widths of all hypertree decompositions of $H$. It has been proved in Adler et al. 2007] that $\operatorname{ghw}(H) \leq \operatorname{hw}(H) \leq 3 \cdot \operatorname{ghw}(H)+1$. This means that for our purposes, hypertree width and generalized hypertree width are equivalent. For simplicity, we will only work with generalized hypertree width.

Observe that for every hypergraph $H$ we have $\operatorname{ghw}(H) \leq \operatorname{tw}(H)+1$. Furthermore, if $H$ is a hypergraph with $V(H) \in E(H)$ we have $\operatorname{ghw}(H)=1$ and $\operatorname{tw}(H)=|V(H)|-1$.

We now give an approximate characterization of (generalized) hypertree width by a game that is a variant of the cops and robber game Seymour and Thomas 1993, which characterizes tree width: In the robber and marshals game on $H$ [Gottlob et al. 2003], a robber plays against $k$ marshals. The marshals move on the hyperedges of $H$, trying to catch the robber. Intuitively, the marshals occupy all vertices of the hyperedges where they are located. In each move, some of the marshals fly in helicopters to new hyperedges. The robber moves on the vertices of $H$. She sees where the marshals will be landing and quickly tries to escape, running arbitrarily fast along paths of $H$, not being allowed to run through a vertex that is occupied by a marshal before and after the flight (possibly by two different marshals). The marshals' objective is to land a marshal via helicopter on a hyperedge containing the vertex occupied by the robber. The robber tries to elude capture. The marshal width $\operatorname{mw}(H)$ of a hypergraph $H$ is the least number $k$ of marshals that have a winning strategy in the robber and marshals game played on $H$ (see Adler 2004 or Gottlob et al. 2003 for a formal definition).

It is easy to see that $\operatorname{mw}(H) \leq \operatorname{ghw}(H)$ for every hypergraph $H$. To win the game on a hypertree of generalized hypertree width $k$, the marshals always occupy guards of a decomposition and eventually capture the robber at a leaf of the tree. Conversely, it can be proved that $\operatorname{ghw}(H) \leq 3 \cdot \operatorname{mw}(H)+1$ Adler et al. 2007.

Observe that for every hypergraph $H$, the generalized hypertree width $\operatorname{ghw}(H)$ is less than or equal to the edge cover number $\rho(H)$ : hypergraph $H$ has a generalized hypertree decomposition consisting of a single bag containing all vertices and having a guard of size $\rho(H)$. On the other hand, the following two examples show that hypertree width and fractional edge cover number are incomparable.

Example 4.1. Consider the class of all graphs that only have disjoint edges. The tree width and hypertree width of this class is 1 , whereas the fractional edge cover number is unbounded. 
Example 4.2. For $n \geq 1$, let $H_{n}$ be the following hypergraph: $H_{n}$ has a vertex $v_{S}$ for every subset $S$ of $\{1, \ldots, 2 n\}$ of cardinality $n$. Furthermore, for every $i \in\{1, \ldots, 2 n\}$ the hypergraph $H_{n}$ has a hyperedge $e_{i}=\left\{v_{S} \mid i \in S\right\}$.

Observe that the fractional edge cover number $\rho^{*}\left(H_{n}\right)$ is at most 2 , because the mapping $x$ that assigns $1 / n$ to every hyperedge $e_{i}$ is a fractional edge cover of weight 2 . Actually, it is easy to see that $\rho^{*}\left(H_{n}\right)=2$.

We claim that the hypertree width of $H_{n}$ is $n$. We show that $H_{n}$ has a hypertree decomposition of width $n$. Let $S_{1}=\{1, \ldots, n\}$ and $S_{2}=\{n+1, \ldots, 2 n\}$. We construct a generalized hypertree decomposition for $H_{n}$ with a tree $T$ having two nodes $t_{1}$ and $t_{2}$. For $i=1,2$, we let $B_{t_{1}}$ contain a vertex $V_{S}$ if and only if $S \cap S_{i} \neq \emptyset$. For each edge $e_{j} \in E\left(H_{n}\right)$, there is a bag of the decomposition that contains $e_{j}$ : if $j \in S_{i}$, then $B_{t_{i}}$ contains every vertex of $e_{j}$. We set the guard $C_{t_{i}}$ to contain every $e_{j}$ with $j \in S_{i}$. It is clear that $\left|C_{t_{i}}\right|=n$ and $C_{t_{i}}$ covers $B_{t_{i}}$ : vertex $v_{S}$ is in $B_{t_{i}}$ only if there is a $j \in S \cap S_{i}$, in which case $e_{j} \in C_{t_{i}}$ covers $v_{S}$. Thus this is indeed a generalized hypertree decomposition of width $n$ for $H_{n}$ and $\operatorname{ghw}\left(H_{n}\right) \leq n$ follows.

To see that $\operatorname{ghw}\left(H_{n}\right)>n-1$, we argue that the robber has a winning strategy against $(n-1)$ marshals in the robber and marshals game. Consider a position of the game where the marshals occupy edges $e_{j_{1}}, \ldots, e_{j_{n-1}}$ and the robber occupies a vertex $v_{S}$ for a set $S$ with $S \cap\left\{j_{1}, \ldots, j_{n-1}\right\}=\emptyset$. Suppose that in the next round of the game the marshals move to the edges $e_{k_{1}}, \ldots, e_{k_{n-1}}$. Let $i \in S \backslash\left\{k_{1}, \ldots, k_{n-1}\right\}$. The robber moves along the edge $e_{i}$ to a vertex $v_{R}$ for a set $R \subseteq\{1, \ldots, 2 n\} \backslash\left\{k_{1}, \ldots, k_{n-1}\right\}$ of cardinality $n$ that contains $i$. If she plays this way, she can never be captured.

For a hypergraph $H$ and a mapping $\gamma: E(H) \rightarrow[0, \infty)$, we let

$$
B(\gamma)=\left\{v \in V(H) \mid \sum_{e \in E(H), v \in e} \gamma(e) \geq 1\right\} .
$$

We may think of $B(\gamma)$ as the set of all vertices "blocked" by $\gamma$. Furthermore, we let $\operatorname{weight}(\gamma)=\sum_{e \in E} \gamma(e)$.

Definition 4.3. Let $H$ be a hypergraph. $A$ fractional hypertree decomposition of $H$ is a triple $\left(T,\left(B_{t}\right)_{t \in V(T)},\left(\gamma_{t}\right)_{t \in V(T)}\right)$, where $\left(T,\left(B_{t}\right)_{t \in V(T)}\right)$ is a tree decomposition of $H$ and $\left(\gamma_{t}\right)_{t \in V(T)}$ is a family of mappings from $E(H)$ to $[0, \infty)$ such that for every $t \in V(T)$ we have $B_{t} \subseteq B\left(\gamma_{t}\right)$.

We call the sets $B_{t}$ the bags of the decomposition and the mappings $\gamma_{t}$ the (fractional) guards.

The width of $\left(T,\left(B_{t}\right)_{t \in V(T)},\left(\gamma_{t}\right)_{t \in V(T)}\right)$ is $\max \left\{\operatorname{weight}\left(\gamma_{t}\right) \mid t \in V(T)\right\}$. The fractional hypertree width fhw $(H)$ of $H$ is the minimum of the widths of the fractional hypertree decompositions of $H$. Equivalently, fhw $(H) \leq r$ if $H$ has a tree decomposition where $\rho^{*}\left(B_{t}\right) \leq r$ for every bag $B_{t}$.

It is easy to see that the minimum of the widths of all fractional hypertree decompositions of a hypergraph $H$ always exists and is rational. This follows from the fact that, up to an obvious equivalence, there are only finitely many tree decompositions of a hypergraph.

Clearly, for every hypergraph $H$ we have

$$
\text { fhw }(H) \leq \rho^{*}(H) \text { and } \operatorname{fhw}(H) \leq \operatorname{ghw}(H) .
$$

Examples 4.1 and 4.2 above show that there are families of hypergraphs of bounded fractional hypertree width, but unbounded fractional edge cover number and unbounded generalized hypertree width.

It is also worth pointing out that for every hypergraph $H$,

$$
\text { fhw }(H)=1 \Longleftrightarrow \operatorname{ghw}(H)=1 \text {. }
$$


To see this, note that if $\gamma: E(H) \rightarrow[0, \infty)$ is a mapping with weight $(\gamma)=1$ and $B \subseteq B(\gamma)$, then $B \subseteq e$ for all $e \in E(H)$ with $\gamma(e)>0$. Thus instead of using $\gamma$ as a guard in a fractional hypertree decomposition, we may use the integral guard $\{e\}$ for any $e \in E(H)$ with $\gamma(e)>0$. Let us remark that $\operatorname{ghw}(H)=1$ if and only if $H$ is acyclic Gottlob et al. 2002 .

\subsection{The robber and army game}

As robbers are getting ever more clever, it takes more and more powerful security forces to capture them. In the robber and army game on a hypergraph $H$, a robber plays against a general commanding an army of $r$ battalions of soldiers. The general may distribute his soldiers arbitrarily on the hyperedges. However, a vertex of the hypergraph is only blocked if the number of soldiers on all hyperedges that contain this vertex adds up to the strength of at least one battalion. The game is then played like the robber and marshals game.

DEFINITION 4.4. Let $H$ be a hypergraph and $r$ a nonnegative real. The robber and army game on $H$ with $r$ battalions (denoted by $\mathrm{RA}(H, r)$ ) is played by two players, the robber and the general. A position of the game is a pair $(\gamma, v)$, where $v \in V(H)$ and $\gamma: E(H) \rightarrow[0, \infty)$ with weight $(\gamma) \leq r$. To start a game, the robber picks an arbitrary $v_{0}$, and the initial position is $\left(0, v_{0}\right)$, where 0 denote the constant zero mapping.

In each round, the players move from the current position $(\gamma, v)$ to a new position $\left(\gamma^{\prime}, v^{\prime}\right)$ as follows: The general selects $\gamma^{\prime}$, and then the robber selects $v^{\prime}$ such that there is a path from $v$ to $v^{\prime}$ in the hypergraph $H \backslash\left(B(\gamma) \cap B\left(\gamma^{\prime}\right)\right)$.

If a position $(\gamma, v)$ with $v \in B(\gamma)$ is reached, the play ends and the general wins. If the play continues forever, the robber wins.

The army width $\operatorname{aw}(H)$ of $H$ is the least $r$ such that the general has winning strategy for the game $\mathrm{RA}(H, r)$.

Again, it is easy to see that $\operatorname{aw}(H)$ is well-defined and rational (observe that two positions $\left(\gamma_{1}, v\right)$ and $\left(\gamma_{2}, v\right)$ are equivalent if $B\left(\gamma_{1}\right)=B\left(\gamma_{2}\right)$ holds $)$.

THEOREM 4.5. For every hypergraph $H$,

$$
\operatorname{aw}(H) \leq \mathrm{fhw}(H) \leq 3 \cdot \operatorname{aw}(H)+2 .
$$

The rest of this subsection is devoted to a proof of this theorem. The proof is similar to the proof of the corresponding result for the robber and marshal game and generalized hypertree width in Adler et al. 2007, which in turn is based on ideas from [Reed 1997; Seymour and Thomas 1993.

Let $H$ be a hypergraph and $\gamma, \sigma: E(H) \rightarrow[0, \infty)$. For a set $W \subseteq V(H)$, we let

$$
\operatorname{weight}(\gamma \mid W)=\sum_{\substack{e \in E(H) \\ e \cap W \neq \emptyset}} \gamma(e) \text {. }
$$

A mapping $\sigma: E(H) \rightarrow[0, \infty)$ is a balanced separator for $\gamma$ if for every connected component $R$ of $H \backslash B(\sigma)$,

$$
\text { weight }(\gamma \mid R) \leq \frac{\operatorname{weight}(\gamma)}{2}
$$

LEMMA 4.6. Let $H$ be a hypergraph with aw $H) \leq r$ for some nonnegative real $r$. Then every $\gamma: E(H) \rightarrow[0, \infty)$ has a balanced separator of weight $r$.

Proof. Suppose for contradiction that $\gamma: E(H) \rightarrow[0, \infty)$ has no balanced separator of weight $r$. We claim that the robber has a winning strategy for the game $\operatorname{RA}(H, r)$. The robber simply maintains the invariant that in every position $(\sigma, v)$ of the game, $v$ is contained in the connected component $R$ of $H \backslash B(\sigma)$ with weight $(\gamma \mid R)>$ weight $(\gamma) / 2$. 
To see that this is possible, let $(\sigma, v)$ be such a position. Suppose that the general moves from $\sigma$ to $\sigma^{\prime}$, and let $R^{\prime}$ be the connected component of $H \backslash B\left(\sigma^{\prime}\right)$ with weight $\left(\gamma \mid R^{\prime}\right)>$ weight $(\gamma) / 2$. Then there must be some $e \in E(H)$ such that $e \cap R \neq \emptyset$ and $e \cap R^{\prime} \neq \emptyset$, because otherwise we had

$$
\operatorname{weight}(\gamma)=\operatorname{weight}(\gamma) / 2+\operatorname{weight}(\gamma) / 2<\operatorname{weight}(\gamma \mid R)+\operatorname{weight}\left(\gamma \mid R^{\prime}\right) \leq \operatorname{weight}(\gamma),
$$

which is impossible. Thus the robber can move from $R$ to $R^{\prime}$ via the edge $e$.

Let $H$ be a hypergraph and $H^{\prime}$ an induced subhypergraph of $H$. Then the restriction of a mapping $\gamma: E(H) \rightarrow[0, \infty)$ to $H^{\prime}$ is the mapping $\gamma^{\prime}: E\left(H^{\prime}\right) \rightarrow[0, \infty)$ defined by

$$
\gamma^{\prime}\left(e^{\prime}\right)=\sum_{\substack{e \in E(H) \\ e \cap V\left(H^{\prime}\right)=e^{\prime}}} \gamma(e) .
$$

Note that weight $\left(\gamma^{\prime}\right) \leq \operatorname{weight}(\gamma)$ and $B\left(\gamma^{\prime}\right)=B(\gamma) \cap V\left(H^{\prime}\right)$. The inequality may be strict because edges with nonempty weight may have an empty intersection with $V\left(H^{\prime}\right)$. Conversely, the canonical extension of a mapping $\gamma^{\prime}: E\left(H^{\prime}\right) \rightarrow[0, \infty)$ to $H$ is the mapping $\gamma: E(H) \rightarrow[0, \infty)$ defined by

$$
\gamma(e)=\frac{\gamma^{\prime}\left(e \cap V\left(H^{\prime}\right)\right)}{\left|\left\{e_{1} \in E(H) \mid e_{1} \cap V\left(H^{\prime}\right)=e \cap V(H)\right\}\right|}
$$

if $e \cap V\left(H^{\prime}\right) \neq \emptyset$ and $\gamma(e)=0$ otherwise. Intuitively, for every $e^{\prime} \in E\left(H^{\prime}\right)$, we distribute the weight of $e^{\prime}$ equally among all the edges $e \in E(H)$ whose intersection with $V\left(H^{\prime}\right)$ is exactly $e^{\prime}$. Note that weight $(\gamma)=$ weight $\left(\gamma^{\prime}\right)$ and $B\left(\gamma^{\prime}\right)=B(\gamma) \cap V\left(H^{\prime}\right)$.

Proof (of Theorem 4.5). Let $H$ be a hypergraph. To prove that $\operatorname{aw}(H) \leq \mathrm{fhw}(H)$, let $\left(T,\left(B_{t}\right)_{t \in V(T)},\left(\gamma_{t}\right)_{t \in V(T)}\right)$ be a fractional hypertree decomposition of $H$ having width fhw $(H)$. We claim that the general has a winning strategy for $\mathrm{RA}(H, r)$. Let $\left(0, v_{0}\right)$ be the initial position. The general plays in such a way that all subsequent positions are of the form $\left(\gamma_{t}, v\right)$ such that $v \in B_{u}$ for some $u \in V\left(T_{t}\right)$. Intuitively, this means that the robber is trapped in the subtree below $t$. Furthermore, in each move the general reduces the height of $t$. He starts by selecting $\gamma_{t_{0}}$ for the root $t_{0}$ of $T$. Suppose the game is in a position $\left(\gamma_{t}, v\right)$ such that $v \in B_{u}$ for some $u \in V\left(T_{t}\right)$. If $u=t$, then the robber has lost the game. So let us assume that $u \neq t$. Then there is a child $t^{\prime}$ of $t$ such that $u \in V\left(T_{t^{\prime}}\right)$. The general moves to $\gamma_{t^{\prime}}$. Suppose the robber escapes to a $v^{\prime}$ that is not contained in $B_{u^{\prime}}$ for any $u^{\prime} \in T_{t^{\prime}}$. Then there is a path from $v$ to $v^{\prime}$ in $H \backslash\left(B\left(\gamma_{t}\right) \cap B\left(\gamma_{t^{\prime}}\right)\right)$ and hence in $H \backslash\left(B_{t} \cap B_{t^{\prime}}\right)$. However, it follows easily from the fact that $\left(T,\left(B_{t}\right)_{t \in T}\right)$ is a tree decomposition of $H$ that every path from a bag in $T_{t^{\prime}}$ to a bag in $T \backslash T_{t^{\prime}}$ must intersect $B_{t} \cap B_{t^{\prime}}$. This proves that aw $(H) \leq \operatorname{fhw}(H)$.

For the second inequality, we shall prove the following stronger claim:

Claim: Let $H$ be a hypergraph with aw $(H) \leq r$ for some nonnegative real $r$. Furthermore, let $\gamma: E(H) \rightarrow[0, \infty)$ such that weight $(\gamma) \leq 2 r+2$. Then there exists a fractional hypertree decomposition of $H$ of width at most $3 r+2$ such that $B(\gamma)$ is contained in the bag of the root of this decomposition. $H$.

Note that for $\gamma=0$, the claim yields the desired fractional hypertree decomposition of

Proof of the claim: The proof is by induction on the cardinality of $V(H) \backslash B(\gamma)$.

By Lemma 4.6, there is a balanced separator of weight at most $r$ for $\gamma$ in $H$. Let $\sigma$ be such a separator, and define $\chi: E(H) \rightarrow[0, \infty)$ by $\chi(e)=\gamma(e)+\sigma(e)$. Then weight $(\chi) \leq 3 r+2$, and $B(\gamma) \cup B(\sigma) \subseteq B(\chi)$. 
If $V(H)=B(\chi)$ (this is the induction basis), then the 1-node decomposition with bag $V(H)$ and guard $\chi$ is a fractional hypertree decomposition of $H$ of width at most $3 r+2$.

Otherwise, let $R_{1}, \ldots, R_{m}$ be the connected components of $H \backslash B(\chi)$. Note that we cannot exclude the case $m=1$ and $R_{1}=V(H) \backslash B(\chi)$.

For $1 \leq i \leq m$, let $e_{i}$ be an edge of $H$ such that $e_{i} \cap R_{i} \neq \emptyset$, and let $S_{i}$ be the unique connected component of $H \backslash B(\sigma)$ with $R_{i} \subseteq S_{i}$. Note that weight $\left(\gamma \mid S_{i}\right) \leq r+1$, because $\sigma$ is a balanced separator for $\gamma$. Let $\chi_{i}: E(H) \rightarrow[0, \infty)$ be defined by

$$
\chi_{i}(e)= \begin{cases}1 & \text { if } e=e_{i} \\ \sigma(e)+\gamma(e) & \text { if } e \neq e_{i} \text { and } S_{i} \cap e \neq \emptyset, \\ \sigma(e) & \text { otherwise. }\end{cases}
$$

Then

$$
\operatorname{weight}\left(\chi_{i}\right) \leq 1+\operatorname{weight}(\sigma)+\operatorname{weight}\left(\gamma \mid S_{i}\right) \leq 2 r+2
$$

and $B\left(\chi_{i}\right) \backslash R_{i} \subseteq B(\chi)$ (as $e_{i}$ cannot intersect any $R_{j}$ with $i \neq j$ ). Let $H_{i}=H\left[R_{i} \cup B\left(\chi_{i}\right)\right]$ and observe that

$$
V\left(H_{i}\right) \backslash B\left(\chi_{i}\right) \subseteq R_{i} \backslash e_{i} \subset R_{i} \subseteq V(H) \backslash B(\gamma)
$$

(the first inclusion holds because $\chi_{i}\left(e_{i}\right)=1$ ). Thus the induction hypothesis is applicable to $H_{i}$ and the restriction of $\chi_{i}$ to $H_{i}$. It yields a fractional hypertree decomposition $\left(T^{i},\left(B_{t}^{i}\right)_{t \in V\left(T^{i}\right)},\left(\gamma_{t}^{i}\right)_{t \in V\left(T^{i}\right)}\right)$ of $H_{i}$ of weight at most $3 r+2$ such that $B\left(\chi_{i}\right)$ is contained in the bag $B_{t_{0}^{i}}^{i}$ of the root $t_{0}^{i}$ of $T^{i}$.

Let $T$ be the disjoint union of $T^{1}, \ldots, T^{m}$ together with a new root $t_{0}$ that has edges to the roots $t_{0}^{i}$ of the $T^{i}$. Let $B_{t_{0}}=B(\chi)$ and $B_{t}=B_{t}^{i}$ for all $t \in V\left(T^{i}\right)$. Moreover, let $\gamma_{t_{0}}=\chi$, and let $\gamma_{t}$ be the canonical extension of $\gamma_{t}^{i}$ to $H$ for all $t \in V\left(T^{i}\right)$.

It remains to prove that $\left(T,\left(B_{t}\right)_{t \in V(T)},\left(\gamma_{t}\right)_{t \in V(T)}\right)$ is a fractional hypertree decomposition of $H$ of width at most $3 r+2$. Let us first verify that $\left(T,\left(B_{t}\right)_{t \in V(T)}\right)$ is a tree decomposition.

- Let $v \in V(H)$. To see that $\left\{v \in V(T) \mid v \in B_{t}\right\}$ is connected in $T$, observe that $\{t \in$ $\left.V\left(T^{i}\right) \mid v \in B_{t_{i}}\right\}$ is connected (maybe empty) for all $i$. If $v \in R_{i}$ for some $i$, then $v \notin V\left(H_{j}\right)=R_{j} \cup B\left(\chi_{j}\right)$ for any $i \neq j$ (as $R_{i}$ and $R_{j}$ are disjoint and we have seen that $\left.B\left(\chi_{j}\right) \backslash R_{j} \subseteq B(\chi)\right)$ and this this already shows that $\left\{t \in V(T) \mid v \in B_{t}\right\}$ is connected. Otherwise, $v \in B\left(\chi_{i}\right) \backslash R_{i} \subseteq B(\chi)=B_{t_{0}}$ for all $i$ such that $v \in V\left(H_{i}\right)$. Again this shows that $\left\{v \in V(T) \mid v \in B_{t}\right\}$ is connected.

- Let $e \in E(H)$. Either $e \subseteq B(\chi)=B_{t_{0}}$, or there is exactly one $i$ such that $e \subseteq R_{i} \cup B\left(\chi_{i}\right)$. In the latter case, $e \subseteq B_{t}$ for some $t \in V\left(T^{i}\right)$.

It remains to prove that $B_{t} \subseteq B\left(\gamma_{t}\right)$ for all $t \in T$. For the root, we have $B_{t_{0}}=B\left(\gamma_{t_{0}}\right)$. For $t \in V\left(T^{i}\right)$, we have $B_{t} \subseteq B\left(\gamma_{t}^{i}\right)=B\left(\gamma_{t}\right) \cap V\left(H_{i}\right) \subseteq B\left(\gamma_{t}\right)$. Finally, note that weight $\left(\gamma_{t}\right) \leq$ $3 r+2$ for all $t \in V(T)$. This completes the proof of the claim.

Remark 4.7. With respect to the difference between hypertree decompositions and generalized hypertree decompositions, it is worth observing that the fractional tree decomposition $\left(T,\left(B_{t}\right)_{t \in V(T)},\left(\gamma_{t}\right)_{t \in V(T)}\right)$ of width at most $3 r+2$ constructed in the proof of the theorem satisfies the following special condition: $B\left(\gamma_{t}\right) \cap \bigcup_{u \in V\left(T_{t}\right)} B_{u} \subseteq B_{t}$ for all $t \in V(T)$. This implies that a hypergraph of fractional hypertree width at most $r$ has a fractional hypertree decomposition of width at most $3 r+2$ that satisfies the special condition.

\subsection{Finding decompositions}

For the algorithmic applications, it is essential to have algorithms that find fractional hypertree decompositions of small width. The question is whether for any fixed $r>1$ there is a polynomial-time algorithm that, given a hypergraph $H$ with $\operatorname{fhw}(H) \leq r$, computes 
a fractional hypertree decomposition of $H$ of width at most $r$ or maybe of width at most $f(r)$ for some function $f$. Similarly to hypertree width, one way of obtaining such an algorithm would be through the army and robber game characterization. The idea would be to inductively compute the set of all positions of the game from which the general wins in $0,1, \ldots$ rounds. The problem is that, as opposed to the robber and marshals game, there is no polynomial bound on the number of positions.

Using a different approach (approximately solving the problem of finding balanced separators with small fractional edge cover number), Marx [2010a gave an algorithm that approximates fractional hypertree width in the following sense:

TheOREM 4.8 (| MARX 2010A]). For every $r \geq 1$, there is an $n^{O\left(r^{3}\right)}$ time algorithm that, given a hypergraph with fractional hypertree width at most $r$, finds a fractional hypertree decomposition of width $O\left(r^{3}\right)$.

The main technical challenge in the proof of Theorem 4.8 is finding a separator with bounded fractional edge cover number that separates two sets $X, Y$ of vertices. An approximation algorithm is given in Marx 2010a for this problem, which finds a separator of weight $O\left(r^{3}\right)$ if a separator of weight $r$ exists. This algorithm is used to find balanced separators, which in turn is used to construct a tree decomposition (by an argument similar to the proof of the second part of Theorem 4.5).

It is shown in Marx 2010a Fomin et al. 2009 that deciding whether $H$ has fractional hypertree width $r$ is NP-hard if $r$ is part of the input. However, the more relevant question of whether for every fixed $r$, a fractional hypertree decomposition of width $r$ can be found in polynomial-time (i.e., if the width bound $O\left(r^{3}\right)$ in Theorem 4.8 can be improved to $r$ ) is still open. Given that it is NP-hard to decide whether a hypergraph has generalized hypertree width at most 3 Gottlob et al. 2009, it is natural to expect that a similar hardness result holds for fractional hypertree width as well.

\subsection{Algorithmic applications}

In this section, we discuss how problems can be solved by fractional hypertree decompositions of bounded width. First we give a basic result, which formulates why tree decompositions and width measures are useful in the algorithmic context: CSP can be efficiently solved if we can polynomially bound the number of solutions in the bags. Recall that $H_{I}$ denotes the hypergraph of a CSP instance $I$. If $\left(T,\left(B_{t}\right)_{t \in V(T)}\right)$ is a tree decomposition of $H_{I}$, then $I\left[B_{t}\right]$ denotes the instance induced by bag $B_{t}$, see Definition 3.4 .

LEMma 4.9. There is an algorithm that, given a CSP instance, a hypertree decomposition $\left(T,\left(B_{t}\right)_{t \in V(T)}\right)$ of $H_{I}$, and for every $t \in V(t)$ a list $L_{t}$ of all solutions of $I\left[B_{t}\right]$, decides in time $C \cdot\|I\|^{O(1)}$ if $I$ is satisfiable (and computes a solution if it is), where $C:=\max _{t \in V(T)}\left|L_{t}\right|$.

Proof. Define $V_{t}:=\bigcup_{t \in V\left(T_{t}\right)} B_{t}$. For each $t \in V(T)$, our algorithm constructs the list $L_{t}^{\prime} \subseteq L_{t}$ of those solutions of $I\left[B_{t}\right]$ that can be extended to a solution of $I\left[V_{t}\right]$. Clearly, $I$ has a solution if and only if $L_{t_{0}}^{\prime}$ is not empty for the root $t_{0}$ of the tree decomposition.

The algorithm proceeds in a bottom-up manner: when constructing the list $L_{t}^{\prime}$, we assume that for every child $t^{\prime}$ of $t$, the lists $L_{t^{\prime}}^{\prime}$ are already available. If $t$ is a leaf node, then $V_{t}=B_{t}$, and $L_{t}^{\prime}=L_{t}$. Assume now that $t$ has children $t_{1}, \ldots, t_{k}$. We claim that a solution $\alpha$ of $I\left[B_{t}\right]$ can be extended to $I\left[V_{t}\right]$ if and only if for each $1 \leq i \leq k$, there is a solution $\alpha_{i}$ of $I\left[V_{t_{i}}\right]$ that is compatible with $\alpha$ (that is, $\alpha$ and $\alpha_{i}$ assign the same values to the variables in $B_{t} \cap V_{t_{i}}=B_{t} \cap B_{t_{i}}$ ). The necessity of this condition is clear: the restriction of a solution of $I\left[V_{t}\right]$ to $V_{t_{i}}$ is clearly a solution of $I\left[V_{t_{i}}\right]$. For sufficiency, suppose that the solutions $\alpha_{i}$ exist for every child $t_{i}$. They can be combined to an assignment $\alpha^{\prime}$ on $V_{t}$ extending $\alpha$ in a well-defined way: every variable $v \in V_{t_{i}} \cap V_{t_{j}}$ is in $B_{t}$, thus $\alpha_{i}(v)=\alpha_{j}(v)=\alpha(v)$ follows for 
such a variable. Now $\alpha^{\prime}$ is a solution of $I\left[V_{t}\right]$ : for each constraint of $I\left[V_{t}\right]$, the variables of the constraint are contained either in $B_{t}$ or in $V_{t_{i}}$ for some $1 \leq i \leq k$, thus $\alpha$ or $\alpha_{i}$ satisfies the constraint, implying that $\alpha^{\prime}$ satisfies it as well.

Therefore, $L_{t}^{\prime}$ can be determined by first enumerating every solution $\alpha \in L_{t}$, and then for each $i$, checking whether $L_{t_{i}}^{\prime}$ contains an assignment $\alpha_{i}$ compatible with $\alpha$. This check can be efficiently performed the following way. Recall that solutions $\alpha$ and $\alpha_{i}$ are compatible if their restriction to $B_{t} \cap B_{t_{i}}$ is the same assignment. Therefore, after computing $L_{t_{i}}^{\prime}$, we restrict every $\alpha_{i} \in L_{t_{i}}^{\prime}$ to $B_{t} \cap B_{t_{i}}$ and store these restrictions in a trie data structure for easy membership tests. Then to check if there is an $\alpha_{i} \in L_{t_{i}}^{\prime}$ compatible with $\alpha$, all we need to do is to check if the restriction of $\alpha$ to $B_{t} \cap B_{t_{i}}$ is in the trie corresponding to $L_{t_{i}}^{\prime}$, which can be checked in $\|I\|^{O(1)}$. Thus $L_{t}^{\prime}$ (and the corresponding trie structure) can be computed in time $\left|L_{t}\right| \cdot\|I\|^{O(1)}$. As every other part of the algorithm can be done in time $\|I\|^{O(1)}$, it follows that the total running time can be bounded by $C \cdot\|I\|^{O(1)}$. Using standard bookkeeping techniques, it is not difficult to extend the algorithm such that it actually returns a solution if one exists.

Lemma 4.9 tells us that if we have a tree decomposition where we can give a polynomial bound on the number of solutions in the bags for some reason (and we can enumerate all these solutions), then the problem can be solved in polynomial time. Observe that in a fractional hypertree decomposition every bag has bounded fractional edge cover number and hence Theorem 3.5 can be used to enumerate all the solutions. It follows that if a fractional hypertree decomposition of bounded width is given in the input, then the problem can be solved in polynomial time. Moreover, if we know that the hypergraph has fractional hypertree width at most $r$ (but no decomposition is given in the input), then we can use brute force to find a fractional hypertree decomposition of width at most $r$ by trying every possible decomposition and then solve the problem in polynomial time. This way, the running time is polynomial in the input size times an (exponential) function of the number of variables. This immediately shows that if we restrict CSP to a class of hypergraphs whose fractional hypertree width is at most a constant $r$, then the problem is fixed-parameter tractable parameterized by the number of variables. To get rid of the exponential factor depending on the number of variables and obtain a polynomial-time algorithm, we can replace the brute force search for the decomposition by the approximation algorithm of Theorem 4.8 Marx 2010a.

THEOREM 4.10. Let $r \geq 1$. Then there is a polynomial-time algorithm that, given a CSP instance I of fractional hypertree width at most $r$, decides if $I$ is satisfiable (and computes a solution if it is).

Proof. Let $I$ be a CSP instance of fractional hypertree width at most $r$, and let $\left(T,\left(B_{t}\right)_{t \in V(T)},\left(\gamma_{t}\right)_{t \in V(T)}\right)$ be the fractional hypertree decomposition of $\mathcal{H}_{I}$ of width $O\left(r^{3}\right)$ computed by the algorithm of Theorem 4.8. By the definition, the hypergraph of $I\left[B_{t}\right]$ has fractional edge cover number $O\left(r^{3}\right)$ for every bag $B_{t}$. Thus by Theorem 3.5 , the list $L_{t}$ of the solutions of $I\left[B_{t}\right]$ has size at most $\|I\|^{O\left(r^{3}\right)}$ and can be determined in time $\|I\|^{O\left(r^{3}\right)}$. Therefore, we can find a solution in time $\|I\|^{O\left(r^{3}\right)}$ using the algorithm of Lemma 4.9 . $\square$

In the remainder of this section, we sketch further algorithmic applications of fractional hypertree decompositions. As these results follow from our main results with fairly standard techniques, we omit a detailed and technical discussion.

It is has been observed by Feder and Vardi [1998] that constraint satisfaction problems can be described as homomorphism problems for relational structures (see Feder and Vardi 1998 or Grohe 2007 for definitions and details). A homomorphism from a structure $A$ to a structure $B$ is a mapping from the domain of $A$ to the domain of $B$ that preserves membership in all relations. With each structure $A$ we can associate a hypergraph $H_{A}$ whose 
vertices are the elements of the domain of $A$ and whose hyperedges are all sets $\left\{a_{1}, \ldots, a_{k}\right\}$ such that $\left(a_{1}, \ldots, a_{k}\right)$ is a tuple in some relation of $A$. For every class $\mathcal{H}$ of hypergraphs, we let $\operatorname{Hom}(\mathcal{H})$ be the problem of deciding whether a structure $A$ with $H_{A} \in \mathcal{H}$ has a homomorphism to a structure $B$. As an immediate corollary to Theorem 4.10, we obtain:

COROLLARY 4.11. Let $\mathcal{H}$ be a class of hypergraphs of bounded fractional hypertree width. Then $\operatorname{Hom}(\mathcal{H})$ is solvable in polynomial time.

An embedding is a homomorphism that is one-to-one. Note that there is an embedding from a structure $A$ to a structure $B$ if and only if $B$ has a substructure isomorphic to $A$. Analogously to $\operatorname{Hom}(\mathcal{H})$, we define the problem $\operatorname{EmB}(\mathcal{H})$ of deciding whether a structure $A$ with $H_{A} \in \mathcal{H}$ has an embedding into a structure $B$. Observe that $\operatorname{EmB}(\mathcal{H})$ is NPcomplete even if $\mathcal{H}$ is the class of paths, which has fractional hypertree width 1 , because the Hamiltonian Path problem is a special case. However, we obtain a fixed-parameter tractability result for $\operatorname{EMB}(\mathcal{H})$ parameterized by the size $\|A\|$ of the input structure $A$ :

THEOREM 4.12. Let $\mathcal{H}$ be a class of hypergraphs of bounded fractional hypertree width. Then $\operatorname{EmB}(\mathcal{H})$ parameterized by the size of the input structure $A$ is fixed-parameter tractable. More precisely, there is an algorithm that, given a structure $A$ with $H_{A} \in \mathcal{H}$ and a structure $B$, decides if there is an embedding of $A$ into $B$ in time $2^{\|A\|^{O(1)}}\|B\|^{O(1)}$.

This follows from Corollary 4.11 with Alon, Yuster, and Zwick's Alon et al. 1995 color coding technique.

In some situations it is necessary to not only decide whether a CSP-instance has a solution or to compute one solution, but to enumerate all solutions. As the number of solutions may be exponential in the instance size, we can rarely expect a polynomial-time algorithm for this problem. Instead, we may ask for a polynomial-delay algorithm, which is required to compute the first solution in polynomial time and after returning a solution is required to return the next solution (or determine that no other solution exists) in polynomial time. Polynomial-delay algorithms for CSPs have been studied more systematically in Bulatov et al. 2012.

THEOREM 4.13. Let $r \geq 1$. Then there is a polynomial-delay algorithm that, given a CSP instance I of fractional hypertree width at most $r$, enumerates all solutions of $I$.

Proof (SKeTCH). Given an instance $I$, the algorithm first computes a fractional hypertree decomposition $\left(T,\left(B_{t}\right)_{t \in V(T)},\left(\gamma_{t}\right)_{t \in V(T)}\right)$ of $\mathcal{H}_{I}$ of width $O\left(r^{3}\right)$ using the algorithm of Theorem 4.8. Then it orders the nodes of $T$ by a preorder traversal and then orders the variables of $I$ in such a way that if $v \in B_{t}$ and $w \in B_{u} \backslash B_{t}$ and $t$ comes before $u$ in the preorder traversal, $v$ is smaller than $w$. For every node $t$ of $T$ the algorithm computes a list of all solutions for $I\left[B_{t}\right]$ and sorts it lexicographically. It is easy to modify the algorithm of Lemma 4.9 to compute the lexicographically first solution and, for every given solution, the lexicographically next solution.

A problem closely related to the problem of enumerating all solutions to a given CSPinstance is the problem of computing the answer for a conjunctive query in a relational database (see Kolaitis and Vardi 1998). To be precise, answering conjunctive queries is equivalent to computing projections of solution sets of CSP-instances to a given subset $V^{\prime}$ of variables. With each conjunctive query, we can associate hypergraph in a similar way as we did for CSP-instances. Then answering queries with hypergraphs in $\mathcal{H}$ is equivalent to computing projections of solution sets of $\operatorname{CsP}(\mathcal{H})$-instances. We define the fractional hypertree width of a conjunctive query to be the fractional hypertree width of its hypergraph. 
THEOREM 4.14. Let $r \geq 1$. Then there is a polynomial-delay algorithm that, given a conjunctive query $Q$ of fractional hypertree width at most $r$ and a database instance $D$, computes the answer $Q(D)$ of $Q$ in $D$.

The proof is a straightforward refinement of the proof of the previous theorem.

\section{CONCLUSIONS}

In this paper we have considered structural properties that can make a constraint satisfaction problem polynomial-time solvable. Previously, bounded hypertree width was the most general such property. Answering an open question raised in Chen and Dalmau 2005. Cohen et al. 2008; Gottlob et al. 2005: Grohe 2007, we have identified a new class of polynomial-time solvable CSP instances: instances having bounded fractional edge cover number. This result suggests the definition of fractional hypertree width, which is always at most as large as the hypertree width (and in some cases much smaller). It turns out that CSP is polynomial-time solvable for instances having bounded fractional hypertree width, if the hypertree decomposition is given together with the instance. This immediately implies that CSP is fixed-parameter tractable parameterized by the number of variables for hypergraphs with bounded fractional hypertree width. Furthermore, together with the algorithm of Marx 2010a finding approximate fractional hypertree decompositions, it also follows that CSP is polynomial-time solvable for this class. Currently, bounded fractional hypertree width is the most general known structural property that makes CSP polynomial-time solvable.

The most natural open question we leave open regarding fractional hypertree width is whether for every fixed $r$ there is a polynomial-time algorithm that decides if a hypergraph has fractional hypertree width at most $r$ (and if so, constructs a decomposition). As the analogous problem for generalized hypertree width is NP-hard for $r=3$, we expect this to be the case for fractional hypertree width as well.

Another open question is whether there are polynomial-time solvable or fixed-parameter tractable families of CSP instances having unbounded fractional hypertree width. Very recently, Marx 2010b showed that, under suitable complexity assumptions, bounded submodular width (a condition strictly more general that bounded fractional hypertree width) exactly characterizes the fixed-parameter tractability of the problem. However, the exact condition characterizing polynomial-time solvability is still an open problem. In light of the results of Marx 2010b], one needs to understand the complexity of the problem for classes that have bounded submodular width, but unbounded fractional hypertree width.

\section{REFERENCES}

AdLER, I. 2004. Marshals, monotone marshals, and hypertree-width. Journal of Graph Theory 47, 275-296.

Adler, I., Gottlob, G., And Grohe, M. 2007. Hypertree width and related hypergraph invariants. Eur. J. Comb. 28, 8, 2167-2181.

Alon, N., Yuster, R., And Zwick, U. 1995. Color-coding. Journal of the ACM 42, 844-856.

Atserias, A., Grohe, M., And Marx, D. 2008. Size bounds and query plans for relational joins. In 49th Annual IEEE Symposium on Foundations of Computer Science (FOCS 2008). 739-748.

Berge, C. 1976. Graphs and Hypergraphs. North Holland.

Bulatov, A. A. 2006. A dichotomy theorem for constraint satisfaction problems on a 3-element set. $J$. $A C M \quad 53,1,66-120$.

Bulatov, A. A. 2011. Complexity of conservative constraint satisfaction problems. ACM Trans. Comput. Log. 12, 4, 24.

Bulatov, A. A., Dalmau, V., Grohe, M., and Marx, D. 2012. Enumerating homomorphisms. J. Comput. Syst. Sci. 78, 2, 638-650.

Bulatov, A. A., Krokhin, A. A., And Jeavons, P. 2001. The complexity of maximal constraint languages. In Proceedings of the 33rd ACM Symposium on Theory of Computing (STOC 2001). 667-674. 
Chen, H. and Dalmau, V. 2005. Beyond hypertree width: Decomposition methods without decompositions. In Principles and Practice of Constraint Programming (CP 2005), P. van Beek, Ed. Lecture Notes in Computer Science Series, vol. 3709. Springer Berlin / Heidelberg, 167-181.

Chen, H. And Grohe, M. 2010. Constraint satisfaction with succinctly specified relations. Journal of Computer System Sciences 76, 847-860.

Chung, F., Frankl, P., Graham, R., and Shearer, J. 1986. Some intersection theorems for ordered sets and graphs. Journal of Combinatorial Theory, Series A 43, 23-37.

Cohen, D. A., Jeavons, P., And Gyssens, M. 2008. A unified theory of structural tractability for constraint satisfaction problems. J. Comput. Syst. Sci. 74, 5, 721-743.

Dalmau, V., Kolaitis, P. G., And Vardi, M. Y. 2002. Constraint satisfaction, bounded treewidth, and finite-variable logics. In Proceedings of the 8th International Conference on Principles and Practice of Constraint Programming (CP 2002), P. V. Hentenryck, Ed. Lecture Notes in Computer Science Series, vol. 2470. Springer-Verlag, 310-326.

Dechter, R. 2003. Constraint Processing. Morgan Kaufmann.

Dechter, R. and Pearl, J. 1989. Tree clustering for constraint networks. Artificial Intelligence 38, 353366.

FAGin, R. 1983. Degrees of acyclicity for hypergraphs and relational database schemes. Journal of the ACM 30, 3, 514-550.

Feder, T. AND VARDi, M. 1998. The computational structure of monotone monadic SNP and constraint satisfaction: A study through datalog and group theory. SIAM Journal on Computing 28, 57-104.

Fomin, F. V., Golovach, P. A., And Thilikos, D. M. 2009. Approximating acyclicity parameters of sparse hypergraphs. In 26th International Symposium on Theoretical Aspects of Computer Science (STACS 2009), S. Albers and J.-Y. Marion, Eds. Leibniz International Proceedings in Informatics (LIPIcs) Series, vol. 3. Schloss Dagstuhl-Leibniz-Zentrum fuer Informatik, Dagstuhl, Germany, 445-456.

Freuder, E. 1990. Complexity of $k$-tree structured constraint satisfaction problems. In Proceedings of the 8th National Conference on Artificial Intelligence. 4-9.

FriedGut, E. AND KAHN, J. 1998. On the number of copies of a hypergraph in another. Israel Journal of Mathematics 105, 251-256.

Gottlob, G., Grohe, M., Musliu, N., Samer, M., and Scarcello, F. 2005. Hypertree decompositions: Structure, algorithms, and applications. In Graph-Theoretic Concepts in Computer Science (WG 2005), D. Kratsch, Ed. Lecture Notes in Computer Science Series, vol. 3787. Springer Berlin / Heidelberg, 1-15.

Gottlob, G., Lee, S. T., Valiant, G., and Valiant, P. 2012. Size and treewidth bounds for conjunctive queries. J. ACM 59, 3, 16:1-16:35.

Gottlob, G., Leone, N., And Scarcello, F. 2000. A comparison of structural CSP decomposition methods. Artif. Intell. 124, 2, 243-282.

Gottlob, G., Leone, N., and Scarcello, F. 2002. Hypertree decompositions and tractable queries. Journal of Computer and System Sciences 64, 579-627.

Gottlob, G., Leone, N., And Scarcello, F. 2003. Robbers, marshals, and guards: Game theoretic and logical characterizations of hypertree width. Journal of Computer and System Sciences 66, 775-808.

Gottlob, G., Miklós, Z., And Schwentick, T. 2009. Generalized hypertree decompositions: NP-hardness and tractable variants. J. ACM 56, 6 .

Grone, M. 2007. The complexity of homomorphism and constraint satisfaction problems seen from the other side. J. ACM 54, 1.

Grohe, M. And Marx, D. 2006. Constraint solving via fractional edge covers. In Proceedings of the seventeenth annual ACM-SIAM symposium on Discrete algorithm (SODA 2006). ACM Press, New York, NY, USA, 289-298.

Grohe, M., Schwentick, T., And Segoufin, L. 2001. When is the evaluation of conjunctive queries tractable? In Proceedings of the 33rd ACM Symposium on Theory of Computing (STOC 2001). 657666.

Impagliazzo, R., Paturi, R., And Zane, F. 2001. Which problems have strongly exponential complexity? J. Comput. System Sci. 63, 4, 512-530.

Jeavons, P., Cohen, D. A., and Gyssens, M. 1997. Closure properties of constraints. Journal of the $A C M$ 44, 4, 527-548.

Kolaitis, P. And VArdi, M. 1998. Conjunctive-query containment and constraint satisfaction. In Proceedings of the 17th ACM Symposium on Principles of Database Systems (PODS 1998). 205-213.

Marx, D. 2008. Closest substring problems with small distances. SIAM Journal on Computing 38, 4, 1382-1410. 
Marx, D. 2010a. Approximating fractional hypertree width. ACM Trans. Algorithms 6, 2, 1-17.

MARX, D. 2010b. Tractable hypergraph properties for constraint satisfaction and conjunctive queries. In Proceedings of the 42nd ACM Symposium on Theory of Computing (STOC 2010). 735-744.

MarX, D. 2011. Tractable structures for constraint satisfaction with truth tables. Theory of Computing Systems 48, 444-464.

Ngo, H. Q., Porat, E., RÉ, C., And Rudra, A. 2012. Worst-case optimal join algorithms. In Proceedings of the 31st symposium on Principles of Database Systems (PODS 2012). ACM, New York, NY, USA, $37-48$.

Reed, B. 1997. Tree width and tangles: A new connectivity measure and some applications. In Surveys in Combinatorics, R. Bailey, Ed. LMS Lecture Note Series Series, vol. 241. Cambridge University Press, $87-162$.

Rhadakrishnan, J. Entropy and counting. Available at http://www.tcs.tifr.res.in/ jaikumar/mypage.html.

Schaefer, T. 1978. The complexity of satisfiability problems. In Proceedings of the 10th ACM Symposium on Theory of Computing (STOC 1978). 216-226.

Schrijver, A. 2003. Combinatorial optimization. Polyhedra and efficiency. Algorithms and Combinatorics Series, vol. 24. Springer, Berlin.

Seymour, P. And Thomas, R. 1993. Graph searching and a min-max theorem for tree-width. Journal of Combinatorial Theory, Series B 58, 22-33.

YANNAKAKIS, M. 1981. Algorithms for acyclic database schemes. In 7th International Conference on Very Large Data Bases (VLDB 1981). 82-94. 